\title{
Intra-articular therapy with tumor necrosis factor- $\alpha$ antagonists: an update
}

\author{
S. Bello, C. Bonali, L. Serafino, C. Rotondo, N. Terlizzi, G. Lapadula \\ Rheumatology Unit, Policlinico Hospital, University of Bari, Italy
}

\begin{abstract}
SUMMARY
The aim of the study was to review from the present literature the intra-articular (IA) use of the TNF-blocking drugs. A total of 28 papers about this topic were found through a search in PubMed; the first publication's date was July 2003.

These studies include a total of 214 patients affected by 12 different joint diseases that reported a total of 1046 intra-articular therapies carried out in 10 different joint sites. Infliximab and etanercept were the most widely used medications. The safety of this treatment clearly emerges from our analysis, while more difficult was the evaluation of its efficacy. Nevertheless we deduced an ideal patient profile that may better respond to the IA anti-TNF treatment.
\end{abstract}

Key words: intra-articular therapy; sieronegative spondyloarthritis; anti-TNF agents; refractory monoarthritis.

Reumatismo, 2013; 65 (6): 257-263

\section{INTRODUCTION}

$\mathrm{M}$ onoarthritis not responding to systemic treatment is not infrequent in chronic inflammatory rheumatisms. A possible rational approach could be to use locally the medications which are effective in the same disease through systemic administration. TNF-blocking agents are among these medications.

There are, however, many unanswered questions, including those on the safety and efficacy of the treatment, the type of patient who could benefit more from therapy, and the preferred joint to inject. In addition, the type of biological to choose, its administration schedule, and how to evaluate its effect are also to be considered. In order to try to answer some of these questions, we consulted the available literature on the subject.

\section{Search methodology}

We searched PubMed using the terms (intra-articular anti-TNF or intraarticular anti-TNF), (intra-articular infliximab or intraarticular infliximab), (intra-articular etanercept or intraarticular etanercept), (intra-articular adalimumab or intraarticular adalimumab), (intra-articular golimumab or intraarticular golimumab), (intra-articular certolizumab pegol or intraarticular certolizumab pegol), identifying 27 articles was performed by searching Google Scholar and using the same words. Among the 28 considered studies, one was a review article, 1 was a basic research article, 9 were case reports, 14 were case series, 1 was a case-control study and 2 were randomized and controlled trials. We did not consider the articles written in a language different from English and Italian, the articles published as images or as abstracts and comment letters to other articles.

\section{Treated diseases}

Patients with 12 different diagnoses were treated with intra-articular (IA) anti-TNF. They were mostly affected by seronegative spondyloarthritis (SpA, 38.8\%) and rheumatoid arthritis (RA, 38.3\%) but in addition to these, other inflammatory joint diseases, some of which have no indication for the systemic treatment with an anti-TNF therapy (Figure 1) have been also treated. 9 studies regarded patients with rheumatoid arthritis (RA, 82 patients, 38.3\%), 10 papers related to patients with psoriatic $\overline{\text { Corresponding author: }}$ Sandro Bello

Via Napoli, 4 - 72100 Brindisi, Italy E-mail: s.bello1@virgilio.it 
arthritis (PsA, 42 patients, 19.6\%), 3 studies with patients affected by juvenile idiopathic arthritis (JIA, 26 patients, 12.1\%), 4 studies with patients suffering from ankylosing spondylitis (AS, 24 patients, $11.2 \%), 6$ studies where patients have seronegative undifferentiated spondyloarthritis (uSPA; 15 patients, $7.0 \%$ ), 2 studies reporting patients with pigmented villonodular synovitis (PVNS, 3 patients, 1.4\%), 1 study related to patients with erosive osteoarthritis (EOA, 10 patients, $4.7 \%$ ), chronic undifferentiated polyarthritis (uA, 8 patients, $3.7 \%$ ), Behcet's disease (BD), enteropathic arthritis (EpA), reactive arthritis (ReA), sarcoid arthritis (SarA, 1 patient, $0.5 \%$ ). The indication for treatment was the persistence of mono-oligoarthritis despite previous conventional therapies.

\section{Patients'features}

Only in 19 studies (117 of 214 patients) the gender could be distinguished: $67 \mathrm{fe}$ males $(57.3 \%)$ and 50 males $(42.7 \%)$. The average age, inferred for 25 studies (200 patients), resulted of 44.7 years. The average disease duration, obtained from 24 studies and related to 184 patients, was 6.6 years. In 15 studies, related to 144 patients, screening tests were performed to evaluate the safety before starting IA therapy with anti-TNF.

\section{Concomitant therapies}

About 156 out of 214 patients ( $72.9 \%)$ took DMARDs, 28 out of 88 patients $(31.9 \%$, data available from 15 studies) took systemic steroids, 115 out of 187 patients (61.5\% calculated from 24 studies) were previously treated with IA steroids in the affected site. 28 out of 49 patients $(57.1 \%$ derived from 9 studies) were receiving NSAIDs or COXIBs. 7 studies reported the use of a systemic biological DMARD in 39 patients (18.2\%): 5 took infliximab (IFX), 1 abatacept, 4 adalimumab (ADA), 7 etanercept (ETA) and 22 a non specified anti-TNF (1-7).

\section{The joint sites}

The IA therapy with anti-TNF was practiced in 10 different joints. The knee joint was the most considered site (87 patients resulting from 17 out of 18 papers, 97 different joints treated for a total of 198 IA therapies); other locations more frequently

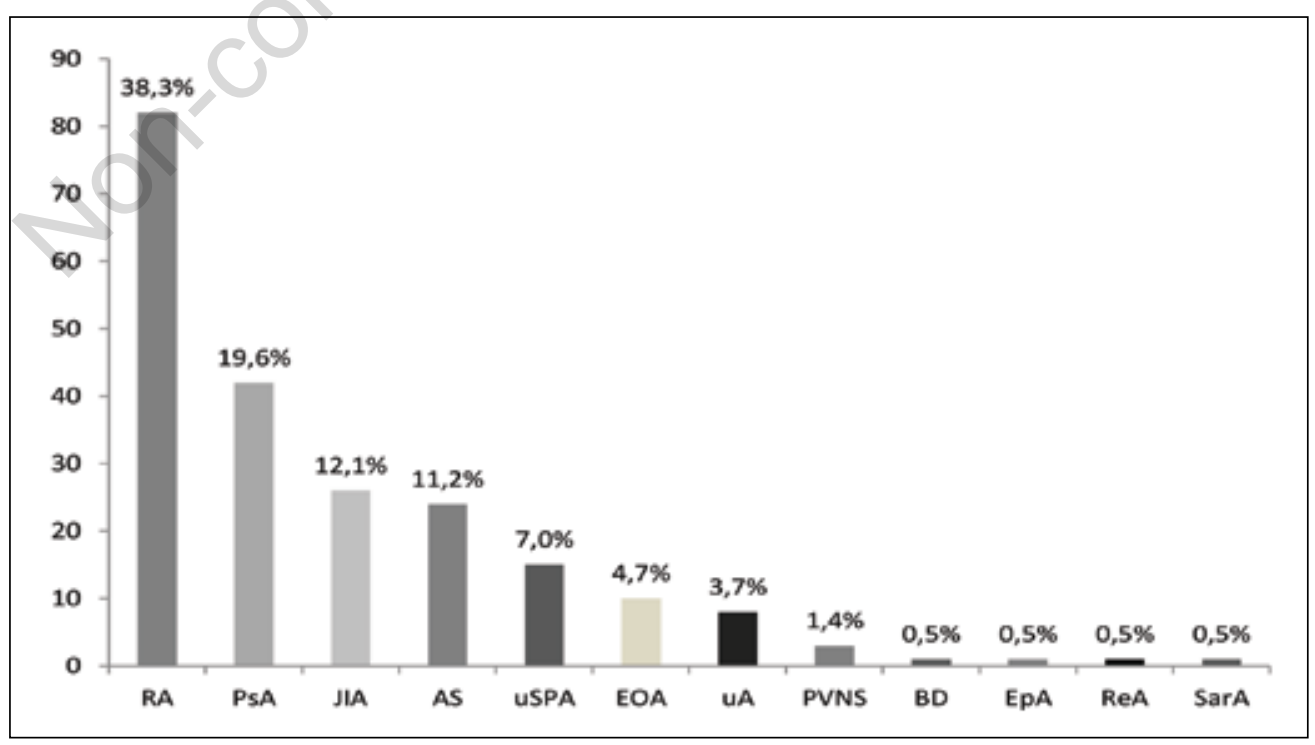

Figure 1 - Patient's distribution for different diseases (RA, rheumatoid arthritis; PsA, psoriatic arthritis; JIA, juvenile idiopathic arthritis; AS, ankylosing spondylitis; uSPA, undifferentiated spondyloarthropathy; EOA, erosive osteoarthritis; UA, undifferentiated arthritis; pigmented villonodular synovitis; BD, Behcet disease; EpA, enteropathic arthritis; ReA, reactive arthritis; SarA, sarcoid arthritis). 


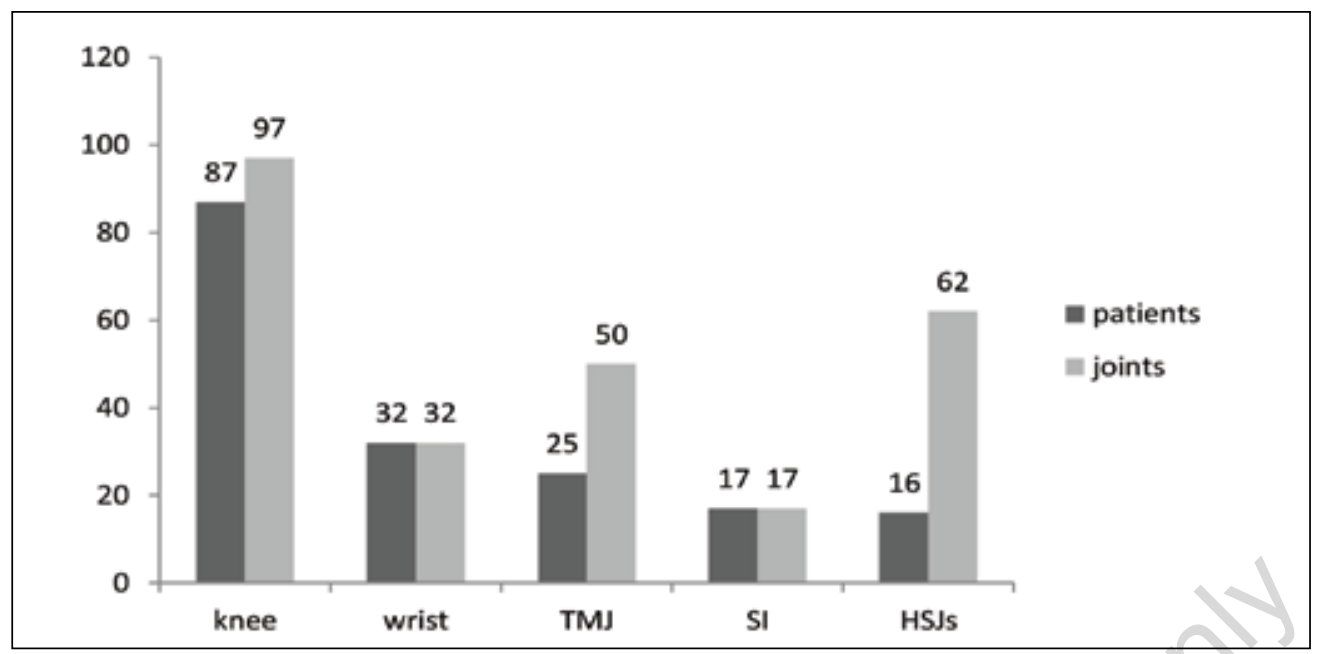

Figure 2 - Number of the treated patients and treated joints, in relation to the sites more numerically considered (TMJ, temporomandibular joint; SI, sacroiliac joint; HSJs, hand small joints).

injected resulted the wrist (32 patients in 5 of 6 papers, 32 different joints for a total of 34 IA therapies), the temporomandibular joint ( 25 patients in 2 papers, 50 different joints for a total of 62 IA therapies), the sacroiliac joint (17 patients in 2 papers, 17 different joints for a total of 49 IA therapies) and the hand small joints (16 patients in 2 papers, 62 different joints for a total of 678 IA therapies) (Figure 2).

The ankles ( 4 patients in 2 articles, 4 joints treated for a total of 4 IA therapies) and the elbows ( 2 patients in 2 papers, available from one of the two papers, 2 joints treated for a total of 2 IA therapies) were less considered.

The infiltration of a popliteal synovial cyst (1 patient, 1 therapy) was reported in one study and this was the only case of local use of anti-TNF in a periarticular structure with inflammatory condition (4).

\section{Anti-TNF agent used}

IFX was used in 15 of the different studies examined (1-5, 8-17), ETA in 8 studies (6, $18-24)$, ADA in $2(25,26)$ while both ADA and ETA were used in 1 study (7). In particular 116 of the 214 patients were treated

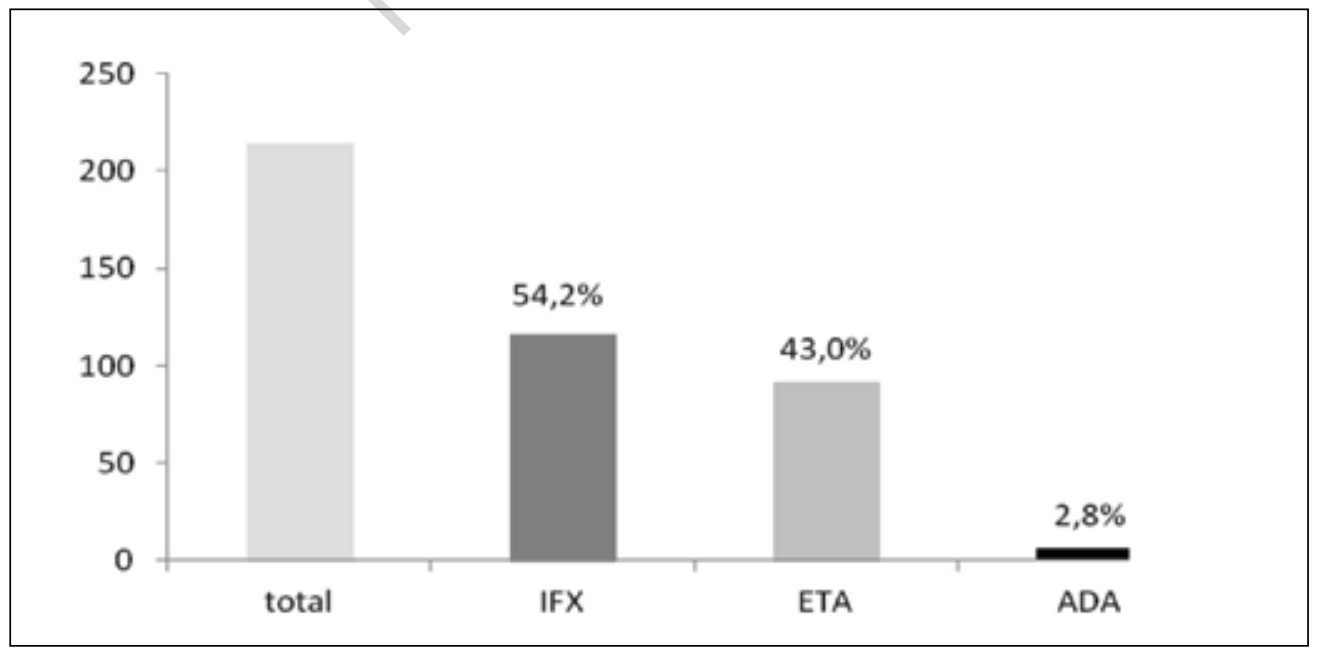

Figure 3 - Patient's distribution for different biological agent. 


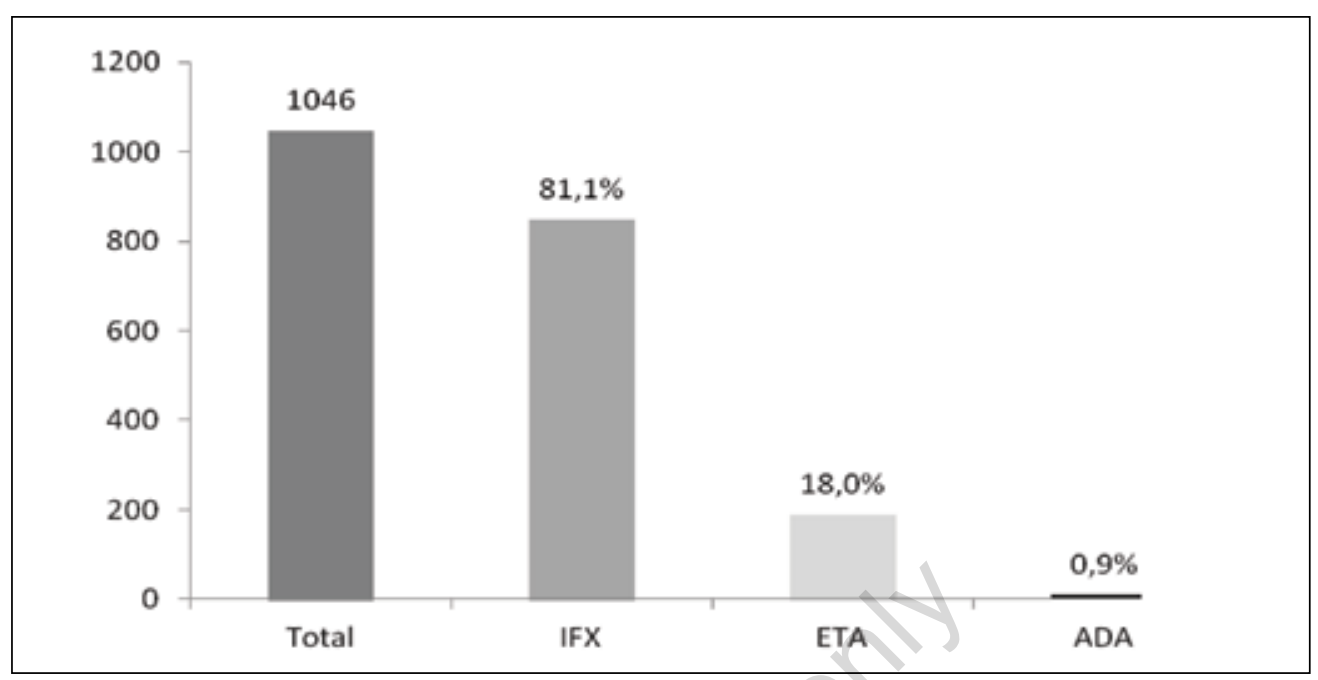

Figure 4 - Distribution of the intra-articular therapies for different biological agent.

with IFX (54.2\%), 92 with ETA (43.0\%) and 6 with ADA (2.8\%). The description of IA use of golimumab and certolizumab was not found (Figure 3).

\section{Number of intra-articular therapies}

A total of 1046 IA therapies were counted in the examined studies, performed with an anti-TNF agent (Figure 4); $848(81.1 \%)$ carried out with IFX, 188 with ETA $(18.0 \%)$ and 10 with ADA (0.9\%). The doses of each drug in the various joints and the number of studies reporting the use of that dose are summarized in Table I. Each patient received from a maximum of 67 to a minimum of 1 IA therapy (average 5.0), while each joint site received from 1 to 12 IA therapies (average 2.5). It is reported the execution of multiple 113 patients, are reported. IA therapies carried out with a mean interval of 7.7 weeks in 16 studies and in relation to 113 patients.

\section{Safety}

Side effects regarding a total of 8 patients were found in 5 studies $(16-18,21,22)$; in particular 2 local side effects were found (an arthritis reactivation of the knee and a thin swelling of the back of the hand) after IA therapy with ETA in the knee and in the wrist and 6 systemic side effects (4 reported as not correlated after IFX, 1 vasovagal reaction after IFX, 1 nonspecific dermatitis after ETA); no particularly serious side effects were seen and showed and all regressed completely. In conclusion it is possible to affirm that the IA therapy with anti-TNF agents is safe, since only

Table I - Different doses of the drugs used in the various joint sites.

\begin{tabular}{|l|l|l|l|}
\hline & IFX $\mathbf{~ m g}$ & ETA mg & ADA mg \\
\hline Knee & $100(10) ; 50(1)$ & $25(4) ; 12.5(2)$ & $40(3)$ \\
\hline Wrist & $50(1) ; 25(1)$ & $25(3) ; 8(1) ; 4(1) ; 2(1)$ & \\
\hline TMJ & $5(2) ; 10(1)$ & & \\
\hline SI & $20(1)$ & $25(1)$ & \\
\hline HSJs & $0.02(1)$ & $8(1) ; 4(1) ; 2(1)$ & \\
\hline Ankle & $25(1)$ & $8(1) ; 4(1) ; 2(1)$ & \\
\hline Elbow & & $25(1) ; 8(1) ; 4(1) ; 2(1)$ & \\
\hline Popliteal synovial cyst & $50(1)$ & & \\
\hline
\end{tabular}

TMJ, temporomandibular joint; SI, sacroiliac joint; HSJs, hand small joints; ( ), number of studies reporting the use of that dosage. 
$3.7 \%$ of the patients reported mild side effects.

\section{Systemic absorption}

Systemic effects after IA administration of the anti-TNF were reported in 12 papers, but only 2 were found to be different from changes in laboratory tests (decrease of the inflammatory markers). The regression of bipolar aphtosis was reported in one study after IFX had been administered in the knees of a patient with Behcet's disease (9). In another one, the regression of a psoriatic plaque on the knee was described $n$ a patient suffering from psoriatic arthritis (4). The efficacy of a new Fv single-chain anti-TNF antibody (ESBA 105) designed for local use and in advanced stage of experimentation was reported in a paper (27). This new drug had similar IFX TNF inhibitory capacity in cell cultures and in vivo was shown to inhibit TNF-induced synovitis in rats with comparable efficacy to IFX. Ex vivo and in vivo studies reported high concentration of ESBA 105 in all the joint tissues and low systemic exposure after local injection $(27,28)$.

\section{Patient evaluation}

A clinimetric evaluation of the patients was carried out in 25 papers while in 24 papers a laboratory evaluation was described. At least one instrumental assessment was performed in 20 studies: ultrasonography (10 papers), MRI (9 papers), synovial biopsy (3 papers), synovial fluid evaluation (2 papers), synovial cytokines evaluation (2 papers), scintigraphy, conventional XR, CT and SPECT (1 paper).

\section{Statistical evaluation}

A statistical evaluation of the results was reported in 11 papers, concerning 180 patients, demonstrating significance for 8 studies related to 137 patients.

\section{Follow up}

The observation period resulted between 3 and 78 weeks, (average 29.9 weeks); the maximum period of remission after treatment was between 168 and 2 weeks (33.6 weeks average).

\section{Effectiveness}

The efficacy of the IA therapy was very difficult to evaluate in the analyzed papers, due to the wide heterogeneity found in the treated diseases, fixed end points, observational periods, timing and number of injections, dosages of the used drugs and different methods in patients assessment. Moreover, the longitudinal evaluation of efficacy case by case was also not possible in all the studies. Although a statistical evaluation related to the majority of patients (180) was used in 11 studies, in most of them the assessment of efficacy was defined by the single investigator more than by statistical evaluation. Only in 4 papers, relatively to 64 patients (29.9\%) a comparison of the results with a control group was reported $(15,16,22,23)$. Instead, one study included a group treated with placebo and then switched to biological therapy, but, in this case, all the comparative evaluations were made during, before and after the IA anti-TNF treatment (6). In another study, the efficacy of IA glucocorticoid therapy was compared to that of IA biologicals performed later (5).

The efficacy of the IA anti-TNF therapy can be deduced through 21 studies, relatively to 142 patients $(68.4 \%)$. This group of responders (R) was compared to the group of patients for which the IA treatment was reported inefficient (non-responders NR, 72 patients, $31.6 \%$ reported in 5 papers). $39.4 \%$ patients in the $\mathrm{R}$ group and $36.1 \%$ in the NR one suffered from RA ( $p=0.77)$, while the percentage of the patients affected from $\mathrm{SpA}$ was significantly higher in the $\mathrm{R}$ group compared to the NR one (50.0\% vs $16.7 \%$; $\mathrm{p}<0.0001)$; in particular AS (15.5\% vs $2.8 \%$; $\mathrm{p}=0.00)$, PsA $(24.6 \%$ vs $9.7 \%$; $\mathrm{p}=0.02)$ and $\mathrm{uSpA}(9.1 \%$ vs $2.8 \%$; $\mathrm{p}=0.18$ ). Patients with EOA, PVNS and two patients with SarA and BD belonged to the $\mathrm{R}$ group while patients with $\mathrm{uA}$ were related to the NR one. Female gender was equally represented in both groups $(54.2 \%$ vs $59.4 \%$; $\mathrm{p}=0.56$ ); while the average age was higher in the R group, (46.4 vs 37.9 years) and the disease duration was lower (6.3 vs 7.9 years). The percentage of patients assuming DMARDs resulted slight- 
ly higher in the $\mathrm{R}$ group (76.0 vs $66.7 \%$; $\mathrm{p}=0.21$ ), instead the rate of patients that assumed a systemic biological agent was lower (11.3 vs 31.9\%; $\mathrm{p}=0.00)$ and the one previously treated with IA steroids (42.9 vs $75 \%$; $\mathrm{p}<0.0001$ ). $72.2 \%$ of the 97 different knees treated with IA anti-TNF belonged to $\mathrm{R}$ group patients $(\mathrm{p}<0.0001)$. A higher percentage in the $\mathrm{R}$ group was made of patients treated with IA ETA (51.4 vs 26.4\%; $\mathrm{p}=0.00$ ), lower resulted the ones treated with IA INF (44.4 vs 73.6\%; $\mathrm{p}<0.0001)$. The total number of IA therapies (952 vs 94), the number of IA therapies for patient (5.8 vs 1.5) and the number of IA therapies per site ( 2.8 vs 1.3$)$ was greater in the $\mathrm{R}$ group. The use of an instrumental evaluation $(92.9$ vs $33.3 \% ; \mathrm{p}<0.0001)$ resulted more frequent in the $\mathrm{R}$ group, whereas the application of a statistical calculation was less frequent $(81.0 \mathrm{vs} 90.3 \%$; $\mathrm{p}=0.1)$. Finally, the maximum interval between 2 following IA therapies (20 vs 12 weeks), the average period of follow-up (35.2 vs 13.8 weeks) and the maximum time of remission (38.0 vs 10.8 weeks) resulted longer in the $\mathrm{R}$ group.

\section{CONCLUSIONS}

A hypothetical patient with persistent monoarthritis refractory to systemic treatment and potentially responsive to IA therapy with anti-TNF may have these features: male or female, 46 years old, suffering from $\mathrm{SpA}$, with disease duration of about 6 years, treated with synthetic DMARD but not with systemic biological DMARD, preferably not previously treated with IA steroids; the knee should be the target joint of this therapy, ETA the drug to be administered and ultrasonography should be the method for instrumental evaluation. This patient profile could be used for further controlled studies to demonstrate the efficacy of IA anti-TNF therapy.

The usefulness of our study is, in our opinion, to have provided through simple calculations a series of data relating to the IA therapy with anti-TNF drugs. The data for the number of patients considered and for some of the obtained results seem encour- aging; however, the percentage of patients included in the only 2 randomized and controlled trials is low $(41 / 214 ; 19: 16 \%)$. The evaluation of effectiveness, complicated by the heterogeneity found in the examined studies and by the difficulty of the direct comparison between the studies themselves, can only provide approximate results.

However it seems that the IA therapy with TNF-blocking agents is not equally effective on all types of chronic inflammatory rheumatism, as it happens also for the systemic administration of these drugs. It thus appears necessary to perform further controlled studies on more selected patients' groups.

\section{REFERENCES}

1. Sakellariou GT, Kakavouli G, Chatzigiannis I. Intraarticular injection of infliximab. J Rheumatol. 2006; 33: 1912-3.

2. Conti F, Ceccarelli F, Priori R, Iagnocco A, Signore A, Valesini G. Intra-articular infliximab in patients with rheumatoid arthritis and psoriatic arthritis with monoarthritis resistant to local glucocorticoids. Clinical efficacy extended to patients on systemic anti-tumour necrosis factor alpha. Ann Rheum Dis. 2008; 67: 178790.

3. Alstergren P, Larsson PT, Kopp S. Successful treatment with multiple intra-articular injections of infliximab in a patient with psoriatic arthritis. Scand J Rheumatol 2008; 37: 155-7.

4. Bello S, Bonali C, Serafino L, Di Giuseppe P, Minosi A, Terlizzi N. Intra-articular therapy with infliximab in psoriatic arthritis: efficacy and safety in refractory monoarthritis. Reumatismo. 2010; 62: 46-50.

5. Stoll ML, Morlandt AB, Teerawattanapong S, Young D, Waite PD, Cron RQ. Safety and efficacy of intra-articular infliximab therapy for treatment-resistant temporomandibular joint arthritis in children: a retrospective study. Rheumatology (Oxford). 2013; 52: 554-9.

6. Fiocco U, Sfriso P, Oliviero F, Roux-Lombard P, Scagliori E, Cozzi L, et al. Synovial effusion and synovial fluid biomarkers in psoriatic arthritis to assess intraarticular tumor necrosis factor-alpha blockade in the knee joint. Arthritis Res Ther. 2010; 12: R148.

7. Haroon M, O'Gradaigh D. Efficacy and safety of combining intra-articular methylprednisolone and anti-TNF agent to achieve prolonged remission in patients with recurrent inflammatory monoarthritis. Joint Bone Spine .2010; 77: 232-4. 
8. Bokarewa M, Tarkowski A. Local infusion of infliximab for the treatment of acute joint inflammation. Ann Rheum Dis. 2003; 62: 783-4.

9. Andonopoulos AP, Meimaris N, Daoussis D, Bounas A, Yiannopoulo G. Intra-articular antitumor necrosis factor alpha antibody in recalcitrant arthritis of Behcet's disease. Clin Exp Rheumatol. 2003; 21: S57-8.

10. Nikas SN, Temekonidis TI, Zikou AK, Argyroupoulo M.I, Efremidis S, Drosos AA. Treatment of resistant rheumatoid arthritis by intra-articular infliximab injections:a pilot study. Ann Rheum Dis. 2004; 63: 102-3.

11. Conti F, Priori R, Chimenti MS, Coari G, Annovazzi A, Valesini G, Signore A. Successful treatment with intraarticular infliximab for resistant knee monoarthritis in a patient with spondyloarthropathy. Arthritis Rheum. 2005; 52: 1224-6.

12. Schatteman L, Gyselbrecht L, De Clercq L, Mielants H. Treatment of refractory inflammatory monoarthritis in ankylosing spondylitis by intra-articular injections of infliximab. $\mathbf{J}$ Rheumatol. 2006; 33: 82-5.

13. Ahern MJ, Campbell DG, Weedon H, Papangelis V, Smith MD. Effect of intra-articular infliximab on synovial membrane pathology in a patient with a seronegative spondyloarthropathy. Ann Rheum Dis. 2008; 67: 133942.

14. O'Shea FD, Haroon N, Salonen DC, Inman RD. Clinical and radiographic response to a local infliximab injection in a patient with chronic sacroiliitis. Nature Clin Pract Rheumatol. 2009; 5: 171-3.

15. Fioravanti A, Fabbroni M, Cerase A, Galeazzi G. Treatment of erosive osteoarthritis of the hands by intra-articular infliximab injections: a pilot study. Rheumatol Int. 2009; 29: 961-5.

16. Van der Bijl AE, Teng YKO, van Oosterhout M, Breedveld FC, Allaart CF, Huizinga TWJ. Efficacy of intraarticular infliximab in patients with chronic or recurrent gonarthritis: a clinical randomized trial. Arthritis Rheum. 2009; 61: 974-8.

17. Levy S, Pilcher J, Heron C, Kiely P. Intraarticular infliximab in DMARD-resistant knee monoarthritis: clinical and ultrasound responses. Int J Clin Rheumatol. 2011; 6: 231-9.

18. Arnold ER, Khanna D, Paulus H, Goodman MP. Acute injection site reaction to intraarticular etanercept administration. Arthritis Rheum. 2003; 48: 2078-9.
19. Hobbs K. Chronic sarcoid arthritis treated with intraarticular etanercept. Arthritis Rheum. 2005; 52: 987-8.

20. Fiocco U, Sfriso P, Oliviero F, Sovran F, Scagliori E, Pagnin E, et al. Intra-articular etanercept treatment for severe diffuse pigmented villonodular knee sinovitis. Reumatismo. 2006; 58: 268-74.

21. Bliddal H, Terslev L, Qvistgaard E, Recke PVD, Holm CC, Danneskiold-Samsoe B, et al. Safety of intra-articular injection of etanercept in small-joint arthritis: an uncontrolled, pilotstudy with independent imaging assessment. Joint Bone Spine. 2006; 73: 714-7.

22. Bliddal H, Terslev L, Qvistgaard E, Koning M, Holm CC, Rogind H, et al. A randomized, controlled study of a single intra-articular injection of etanercept or glucocorticosteroids in patients with rheumatoid arthritis. Scand J Rheumatol. 2006; 35: 341-5.

23. Boesen M, Boesen L, Jensen KE, Cimmino MA, Torp-Pedersen S, Terslev L, et al. Clinical outcome and imaging changes after intraarticular (IA) application of etanercept or methylprednisolone in rheumatoid arthritis:magnetic resonance imaging and ultrasound-Doppler show no effect of IA injections in the wrist after 4 weeks. J Rheumatol. 2008; 35: 584-91.

24. Cui Y, Xiao Z, Shuxia W, Zhenijun Z, Hengguo Z, Liangyi F, et al. Computed tomography guided intra-articular injection of etanercept in the sacroiliac joint is an effective mode of treatment of ankylosing spondylitis. Scand J Rheumatol. 2010; 39: 229-32.

25. Kobak S. Osteonecrosis and monoarticular rheumatoid arthritis treated with intra-articular adalimumab. Mod Rheumatol. 2008; 18: 290-2.

26. Kobak S. Intraarticular adalimumab in a patient with pigmented villonodular synovitis. Rheumatol Int. 2011; 31: 251-4.

27. Urech DM, Feige U, Ewert S, Schlosser V, Ottiger M, Polzer K, et al. Anti-inflammatory and cartilage-protecting effects of an intraarticularly injected anti-TNF\{alpha\} singlechain Fv antibody (ESBA 105) designed for local therapeutic use Ann Rheum Dis. 2010; 69: 443-9.

28. Fisher BAC, Keat A. Should we be using intraarticular tumor necrosis factor blockade in inflammatory monoarthritis? Rheumatology 2006; 33: 1934-5. 